\title{
Artigo/Article
}

\section{Monitoramento da suscetibilidade de populações de Triatoma sordida Stål, 1859 (Hemiptera: Reduviidae) ao inseticida deltametrina, na região Centro-Oeste do Brasil}

Monitoring the susceptibility of Triatoma sordida Stål, 1859 (Hemiptera: Reduviidae) to deltamethrin insecticide, in Central-Western Brazil

\author{
Marcos Takashi Obara ${ }^{1}$, Vanessa Camargo Garbeloto Otrera ${ }^{2}$, Rodrigo Gurgel Gonçalves ${ }^{3}$, Janduhy Pereira dos \\ Santos $^{4}$, Marcelo Santalucia ${ }^{5}$, João Aristeu da Rosa ${ }^{6}$, Paulo Silva de Almeida ${ }^{7}$ e José Maria Soares Barata ${ }^{8}$
}

\section{RESUMO}

Introdução: Populações de Triatoma sordida Stål, 1859 foram investigadas quanto à suscetibilidade à deltametrina. Métodos: Análise por meio de bioensaios por aplicação tópica em 11 populações de T. sordida procedentes dos Estados de Goiás, Mato Grosso e Mato Grosso do Sul. Resultados: As estimativas de $\mathrm{DL}_{50}$ e $\mathrm{RR}_{50}$ demonstraram elevados níveis de suscetibilidade $\left(\mathrm{DL}_{50}<1\right.$ e $\left.\mathrm{RR}_{50}<2\right)$. Entretanto, as análises do coeficiente angular da curva dose resposta revelaram que as populações de triatomíneos dos municípios de Firminópolis/GO, Posse/GO, Poxoréu/MT, Douradina/MS e Aparecida do Taboado/MS apresentam maiores probabilidades de evolução de resistência, portanto, mais propícias a tolerar o tratamento com deltametrina. Conclusões: Detectaram-se pequenas alterações de suscetibilidade e baixos níveis de resistência, porém as alterações temporais de suscetibilidade deverão ser continuamente monitoradas, a fim de nortear adequadamente as ações de controle dos vetores da DC.

Palavras-chaves: Triatomíneos. Resistência. Inseticidas. Deltametrina. Triatoma sordida.

\begin{abstract}
Introduction: Triatoma sordida Stål, 1859 populations were investigated for susceptibility to deltamethrin. Methods: This study aimed to analyze the resistance of 11 populations of insects from the States of Goiás, Mato Grosso and Mato Grosso do Sul by topical application bioassays. Results: The estimated $\mathrm{LD}_{50}$ and $\mathrm{RR}_{50}$ showed high levels of susceptibility $\left(\mathrm{LD}_{50}<1\right.$ and $\mathrm{RR}_{50}$ $<2)$. However, analysis of the slope of the dose response curve showed that the populations of insects in the towns of Firminópolis/GO, Posse/GO, Poxoréu/MT, Douradina/MS and Aparecida do Taboado/MS present great probability of evolving resistance and, thus, are more likely to tolerate treatment with deltamethrin. Conclusions: Small changes in susceptibility and low levels of resistance were detected, but the temporal changes of susceptibility should be continuously monitored to adequately guide the actions of vector-control of DC.
\end{abstract}

Keywords: Triatominae. Resistance. Insecticide. Deltamethrin. Triatoma sordida.

1. Laboratório de Entomologia Médica, Secretaria de Vigilância em Saúde, Ministério da Saúde, Brasília, DF. 2. Laboratório do Núcleo de Pesquisa, Superintendência de Controle de Endemias, Marília, SP. 3. Laboratório de Parasitologia Médica e Biologia de Vetores, Universidade de Brasília, Brasília, DF. 4. Departamento de Estradas de Rodagem, Núcleo de Geoprocessamento, Brasília, DF. 5. Laboratório de Saúde Pública Dr. Giovanni Cysneiros, Secretaria de Estado da Saúde de Goiás, Goiânia, GO. 6. Laboratório de Parasitologia, Faculdade de Ciências Farmacêuticas, Universidade Estadual Paulista, Araraquara, SP. 7. Laboratório Regional de Entomologia, Secretaria de Estado de Saúde, Dourados, MS. 8. Departamento de Epidemiologia, Faculdade de Saúde Pública, Universidade de São Paulo, São Paulo, SP.

Endereço para correspondência: Dr. Marcos Takashi Obara. Lab. Entomologia Médica/CGLAB/SVS/

MS. SCS, Quadra 04, Bloco A, Lote 67/97, 70304-000 Brasília, DF.

Tel: 5561 3213-8272

e-mail: marcos.obara@saude.gov.br

Recebido para publicação em 29/07/2010

Aceito em 21/10/2010

\section{INTRODUÇÃO}

Triatoma sordida Stål, 1859 destaca-se, atualmente, como a espécie de triatomíneo mais capturada no Brasil, predominantemente, em ambiente peridomiciliar, sendo potencial vetor de Trypanosoma cruzi, agente etiológico da doença de Chagas (DC) ${ }^{1-3}$. Nesse ambiente, pode ser encontrado habitando ecótopos, tais como: montes de madeira e de telhas, cercas de madeira, galinheiros e chiqueiros ${ }^{4,5}$. Em ambiente natural, vive, preferencialmente, em cascas de árvores secas ou mortas ${ }^{6}$.

Em maio de 2006, T. sordida foi incriminado como potencial vetor responsável pela ocorrência de sete casos agudos de DC, dos quais dois pacientes evoluíram para óbito, na cidade de Macaúbas, Estado da Bahia, Brasil. A investigação entomológica detectou a presença de colônia intradomiciliar na pia da cozinha. Nesse local, eram armazenados alimentos que, provavelmente, foram contaminados e ingeridos pelos moradores da residência. Essas evidências indicaram que a infecção ocorreu por meio de transmissão oral ${ }^{7}$.

Devidoàinexistência de uma vacina suficientemente segura e eficaz, como também de expectativa de melhoria da progressão clínica da doença entre os portadores crônicos, a estratégia básica de controle da transmissão da DC depende do combate aos vetores por meio de aplicação periódica e sistemática de inseticidas de ação residual, no intra e peridomicílio ${ }^{8}$.

Originalmente, as ações de controle de populações triatomínicas eram realizadas com inseticidas organoclorados. Porém, a partir de 1983, o Programa Nacional de Controle da Doença de Chagas (PNCD) instituiu, no Brasil, o uso de piretróides de síntese em larga escala ${ }^{9,10}$. Certamente, a expansão do uso de inseticidas vem acompanhada pelas constantes questões sobre o potencial de evolução de resistência dos vetores da DC. Nos dias 
atuais, os conhecimentos existentes sobre o perfil de resistência de triatomíneos, além de esparsos, são em geral isolados e, em alguns casos, inexistentes.

Os estudos de monitoramento de suscetibilidade de triatomíneos realizados pelo Centro de Investigaciones en Plagas e Insecticidas (CIPEIN), Buenos Aires, Argentina, têm detectado elevados níveis de resistência aos piretróides deltametrina, $\beta$-cipermetrina e $\beta$-ciflutrina em populações de Triatoma infestans (Klug, 1834) procedentes do norte da Argentina, sul do Brasil e Bolívia ${ }^{11-16}$

Recentemente, no Brasil, avaliações de suscetibilidade de T. sordida registraram níveis de resistência incipiente a deltametrina em cinco populações procedentes dos municípios de Presidente Juscelino, Bocaiúva e Coração de Jesus, Estado de Minas Gerais ${ }^{17}$.

A detecção de alterações na suscetibilidade de populações de T. sordida evidencia a necessidade de novos estudos sobre os níveis de tolerância aos inseticidas piretróides, as quais vêm sendo utilizadas, pelo menos nos últimos vinte anos, nas ações de controle dos vetores da DC no Brasil e em diversas áreas da América Latina.

Nesse contexto, o objetivo desta pesquisa foi analisar os níveis de suscetibilidade de populações de T. sordida procedentes da região Centro-Oeste do Brasil ao piretróide deltametrina, a fim de fornecer parâmetros sobre o perfil de resistência desse vetor. Outro intuito foi subsidiar a implantação de programa de monitoramento de resistência dos vetores da $\mathrm{DC}$, imprescindíveis não só para o Brasil, como também para toda a América Latina.

\section{MÉTODOS}

\section{Triatomíneos para avaliação de resistência}

As populações de triatomíneos foram obtidas a partir de amostras peridomiciliares procedentes dos Estados de Goiás, Mato Grosso e Mato Grosso do Sul. Os insetos foram capturados em áreas endêmicas tratadas com inseticidas, nas quais os programas estaduais de controle da doença de Chagas realizam aplicações contínuas e sistemáticas com inseticidas de ação residual.

A seleção dos municípios, para as atividades de captura, obedeceu aos seguintes critérios: I) a realização de um programa específico de controle de vetores; II) antecedentes históricos de controle de outros vetores dentro da mesma área geográfica; III) uso de inseticidas com finalidades agrícolas na mesma área, onde se localiza os vetores e IV) uso doméstico de inseticidas.

Após o recebimento, cada população foi identificada por meio de chave dicotômica ${ }^{18}$.

\section{População de referência}

Neste trabalho, foi selecionada uma população de referência de T. sordida, cuja criação iniciou-se em 08/03/1982, no insetário do Departamento de Ciências Biológicas da Faculdade de Ciências Farmacêuticas (UNESP-Araraquara), a partir de exemplares procedentes do município de Cordeiros, Estado da Bahia. Os critérios estabelecidos para selecionar a população de referência, para monitoramento de resistência de triatomíneos, estão estabelecidos no protocolo da Organização Mundial da Saúde ${ }^{19}$.

\section{Inseticida}

O inseticida utilizado nos bioensaios foi o piretróide deltametrina [(S) - $\dot{\alpha}$ - ciano - 3 - fenoxibenzil (1R) - cis - 3 - (2,2 - dibromovinil) 2,2 dimetil ciclopropano carboxilato] com grau de pureza de $98,2 \%$, concedido pela Bayer" (Brasil).

\section{Bioensaios}

Foram utilizadas, no mínimo cinco concentrações iniciais do piretróide deltametrina para estimar as taxas de mortalidade entre 0 e $100 \%$. Os ensaios biológicos consistiam na aplicação tópica de $0,2 \mu l$ de solução de deltametrina no dorso do abdômen de ninfas de $1^{\circ}$ estádio entre cinco a sete dias de idade, em jejum. As aplicações foram realizadas utilizando microsseringa de $10 \mu \mathrm{l}$, modelo $1701 \mathrm{~N}$ provida de dispensador com 50 repetições, modelo PB600-1, ambos da Halmilton". Nos insetos do grupo controle, foi aplicado o mesmo volume de acetona PA $\left(\mathrm{CH}_{3}\right)_{2} \mathrm{CO}$, da Vetec".

Para cada concentração, foram utilizadas 20 ninfas, assim o número total de ninfas utilizadas foi de 100 por linhagem. Também foram utilizadas doses intermediárias, a fim de aprimorar os resultados atingidos. As concentrações finais de deltametrina variaram de 0,1 a $3,5 \mu \mathrm{g} / \mathrm{mL}$. Todos os ensaios foram repetidos em laboratório, pelo menos cinco vezes, em diferentes dias.

As aplicações tópicas foram executadas por um único pesquisador, sob microscópio estereoscópico Leica" (Zoom 2000), em ampliação de 10,5X para aumentar a confiabilidade na distribuição do inseticida. Os ensaios biológicos de material capturado em campo foram realizados com ninfas obtidas da primeira e segunda geração (F1 e F2).

A taxa de mortalidade foi observada após $24 \mathrm{~h}$ de exposição do inseticida, por uma única pessoa. As ninfas que atingiram a borda do disco, no período de até 50 segundos, sem alteração nos movimentos de locomoção foram consideradas vivas (normais). Por outro lado, os insetos foram considerados mortos (intoxicados) quando não apresentaram atividade locomotora própria, ou seja, fortes sintomas de desequilíbrio ou totalmente incapazes de andar sozinhos, do centro até a borda de um disco de papel, quando estimulados com a ponta da pinça, durante esse mesmo período.

\section{Análise estatística}

Os dados de mortalidade das populações de T. sordida foram utilizados para estimar a concentração que mata $50 \%\left(\mathrm{DL}_{50}\right)$ e $95 \%$ $\left(\mathrm{DL}_{95}\right)$ das amostras estudadas. $\mathrm{O}$ cálculo da $\mathrm{DL}_{50}$ e $\mathrm{DL}_{95}$ de cada população foi baseado na análise $\mathrm{Probit}^{20} \mathrm{e}$ foi realizado pelo programa Basic Probit Analisys. As doses letais foram expressas em nanograma por ninfa inseto (ng/inseto).

As estimativas de variação das $\mathrm{DL}_{50}$ e $\mathrm{DL}_{95}$ entre as populações foram comparadas por análise de variância (Kruskal-Wallis, $\mathrm{H}$ ) e por testes de Mann-Whitney. Na análise de variância, os resultados dos bioensaios foram testados quanto à hipótese de nulidade de que as estimativas de $\mathrm{DL}_{50}$ e $\mathrm{DL}_{95}$ de cada população eram iguais contra a hipótese alternativa de que pelo menos uma das médias de cada parâmetro era diferente, estabelecendo-se o nível de significância $5 \%$.

O teste Mann-Whitney foi aplicado para calcular os níveis de significância descritivos para diferenças de $\mathrm{DL}_{50}$ e $\mathrm{DL}_{95}$, entre pares sucessivos das médias estudadas.

As $R_{50}$ e $R_{95}$ foram obtidas por meio do quociente da $\mathrm{DL}_{50}$ da população de campo, pela $\mathrm{DL}_{50}$ da linhagem susceptível. O intervalo de confiança de 95\% (IC 95\%) de cada população foi calculado e comparado com a população de referência usando o programa Polo $\mathrm{PC}^{21}$. Os valores da $\mathrm{RR}_{50}$ e $\mathrm{RR}_{95}$ foram considerados significantemente diferentes quando não houve sobreposição dos limites dos intervalos de confiança, no nível de $95 \%$ da população de referência com as amostras de campo. 
Ainda, o coeficiente angular da curva dose resposta foi caracterizado para cada população. Esse parâmetro representa a variação de homogeneidade da população e indica a progressão de resistência e variação genotípica na tolerância a um inseticida. Os menores valores do coeficiente angular da curva indicam populações menos homogêneas, consequentemente com maior probabilidade de seleção de resistência.

\section{RESULTADOS}

A suscetibilidade para deltametrina de onze populações de T. sordida procedentes de diferentes municípios da região central do brasil foi avaliada e comparada com a população de referência (TsREF). As estimativas de $\mathrm{DL}_{50}$ e $\mathrm{DL}_{95}$ da população de referência (TsREF) foram de $0,58 \mathrm{ng} /$ inseto e $1,35 \mathrm{ng} /$ inseto, respectivamente. Nas estimativas de $\mathrm{DL}_{50}$ e $\mathrm{DL}_{95}$ das populações de campo observaramse variações entre 0,61 a $0,86 \mathrm{ng}$ /inseto para os valores $\mathrm{DL}_{50}$ e 1,61 a 3,20ng/inseto para $\mathrm{DL}_{95}$ (Tabela 1).
A análise de variância detectou diferença estatisticamente significativa da $\mathrm{DL}_{50}\left(\mathrm{H}_{11,64}=34,88 ; \mathrm{p}<0,01\right)$ e $\mathrm{DL}_{95}\left(\mathrm{H}_{11,64}=38,03\right.$; $\mathrm{p}<0,01)$ entre as populações analisadas. Quanto às estimativas de $\mathrm{DL}_{50}$, pode se observar que as populações de triatomíneos dos municípios de Guarani do Goiás/GO (TsG), Aparecida do Taboado/ MS (TsA), Douradina/MS (TsD) e Posse/GO (TsPs) apresentaram diferenças significativas com a população de referência (TsREF), conforme mostra a Figura 1. Esses resultados indicam que essas populações exibiram menor suscetibilidade em relação à população de referência e podem tolerar maiores doses de deltametrina. Resultados semelhantes foram obtidos comparando os valores de $\mathrm{DL}_{95}$.

Os resultados estimados para $R_{50}$ confirmaram as diferenças de $\mathrm{DL}_{50}$ e $\mathrm{DL}_{95}$ das amostras estudadas. As populações dos municípios de Guarani do Goiás/GO (TsG), Aparecida do Taboado/MS (TsA), Douradina/MS (TsD) e Posse/GO (TsPs), também, apresentaram diferenças significativas na $\mathrm{RR}_{50}$, quando os limites de confiança foram sobrepostos ao nível de 95\% (Tabela 1). Em relação a $\mathrm{RR}_{95}$, as populações de triatomíneos que apresentaram

TABELA 1 - Distribuição do número de exemplares testados por amostra, $\mathrm{DL}_{50}, \mathrm{DL}_{95}, \mathrm{RR}_{50}$ e $\mathrm{RR}_{95}$ de populações de Triatoma sordida tratadas com aplicação tópica de $0,2 \mu \mathrm{l}$ de deltametrina, na superfície dorsal do abdômen de ninfas de $1^{\circ}$ estádio capturadas, no Brasil, em 2008.

\begin{tabular}{|c|c|c|c|c|c|}
\hline Populações & Número & $\mathrm{DL}_{50} \mathrm{ng} /$ inseto (95\% IC) & $\mathrm{DL}_{95} \mathrm{ng} /$ inseto (95\% IC) & $\mathbf{R R}_{50}(95 \%$ IC $)$ & $\mathbf{R R}_{95}(95 \%$ IC) \\
\hline TsREF & 800 & $0,58(0,54-0,63)$ & $1,35(1,20-1,57)$ & $1,00(0,90-1,10)$ & $1,00(0,82-1,20)$ \\
\hline Campo Grande/MS & 800 & $0,61(0,56-0,67)$ & $1,61(1,39-1,95)$ & $1,05(0,94-1,18)$ & $1,19(1,96-1,47)$ \\
\hline Poxoréu/MT & 880 & $0,64(0,57-0,71)$ & $2,06(1,76-2,56)$ & $1,10(0,96-1,25)$ & $1,52(1,21-1,91)$ \\
\hline Terenos/MS & 560 & $0,69(0,61-0,77)$ & $1,69(1,48-2,03)$ & $1,19(1,04-1,35)$ & $1,24(1,01-1,53)$ \\
\hline Firminópolis/GO & 1.040 & $0,70(0,63-0,78)$ & $2,37(1,99-2,94)$ & $1,20(1,05-1,37)$ & $1,75(1,38-2,21)$ \\
\hline Rochedo/MS & 800 & $0,70(0,63-0,77)$ & $1,73(1,53-2,04)$ & $1,21(1,07-1,36)$ & $1,28(1,05-1,55)$ \\
\hline São Luís de Montes Belos/GO & 700 & $0,71(0,65-0,76)$ & $1,75(1,51-2,18)$ & $1,21(1,08-1,35)$ & $1,29(1,03-1,62)$ \\
\hline Guarani de Goiás/GO & 700 & $0,75(0,70-0,80)$ & $1,55(1,37-1,79)$ & $1,29(1,17-1,42)$ & $1,13(0,94-1,36)$ \\
\hline Aparecida do Taboado/MS & 700 & $0,78(0,70-0,86)$ & $2,60(2,09-3,61)$ & $1,33(1,17-1,51)$ & $1,91(1,42-2,57)$ \\
\hline Douradina/MS & 560 & $0,85(0,78-0,92)$ & $2,17(1,87-2,66)$ & $1,45(1,30-1,62)$ & $1,60(1,28-1,99)$ \\
\hline Posse/GO & 880 & $0,86(0,76-0,96)$ & $3,20(2,70-4,02)$ & $1,48(1,29-1,69)$ & $2,36(1,86-2,99)$ \\
\hline
\end{tabular}

DL: dose letal em nanogramas por inseto, 95\%IC: intervalo de confiança 95\%, RR: razão de resistência, TsREF: população de referência.

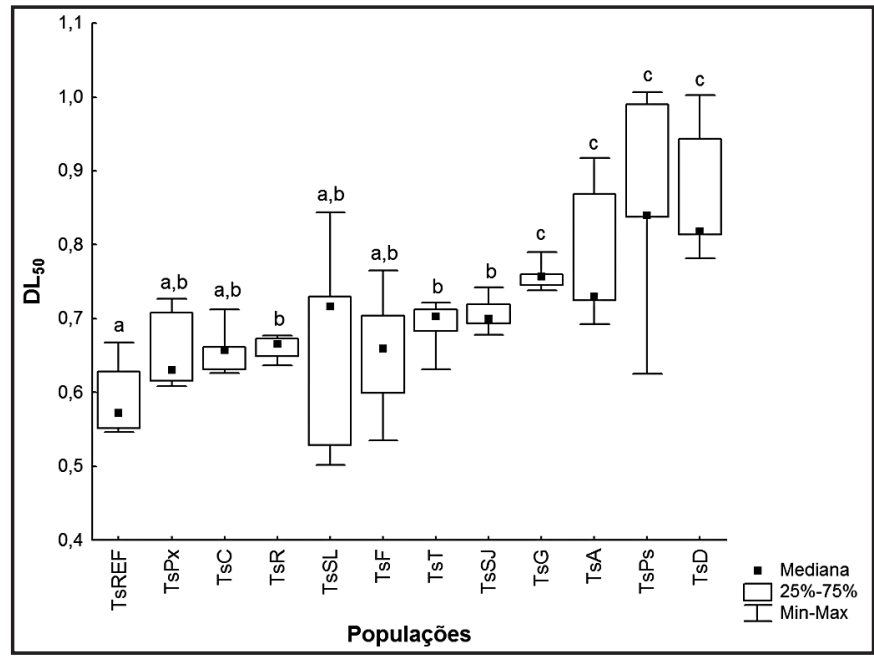

FIGURA 1 - Resultado da $\mathrm{DL}_{50}$ em populações de Triatoma sordida. As letras que não são iguais a população TsRef (a) indicam populações estatisticamente diferentes $(\mathbf{p}<0,01)$ DL: dose letal em nanogramas por inseto.

TsREF: população de referência, TsPx: Poxoréu/MT, TsC: Campo Grande/MS, TsR: Rochedo/MS, TsSL: São Luís de Montes Belos/GO, TsF: Firminópolis/ GO, TsT: Terenos/MS, TsSJ: São José do Povo/MT, TsG: Guarani de Goiás/ GO, TsA: Aparecida do Taboado/MS, TsPs: Posse/GO, TsD: Douradina/MS. 
diferenças significativas com a população de referência pertencem aos municípios de Aparecida do Taboado/MS (TsA), Posse/GO (TsPs) e Douradina/MS (TsD). Apesar das diferenças significativas, os resultados obtidos indicaram baixos níveis de resistência, em todas as amostras analisadas.

Conforme mostram as Figuras $\mathbf{2 A}$ e 2B, as populações com menores taxas de variação na homogeneidade foram oriundas dos municípios de Guarani de Goiás/GO (TsG) e São Luis dos Montes Belos/GO (TsSL). Entretanto, as populações de triatomíneos dos municípios de Firminópolis/GO (TsF), e Posse/ GO (TsPs), mostradas nas Figuras 2C e 2D foram consideradas mais heterogêneas e com maiores chances de tolerar a pressão de tratamento com piretróides.

Como se pode ver na Figura 3A, a população de São José do Povo/MT (TsSJ) mostrou uma variação de inclinação da reta no gráfico muito próxima à população de referência, ao contrário da população de T. sordida do município de Poxoréu/MT (TsPx), mostrada na Figura 3B, que apresentou um valor do coeficiente angular menor que a população de referência, consequentemente possui maior probabilidade de evolução de resistência.

Nas populações de triatomíneos procedentes dos municípios de Campo Grande/MS (TsC), Terenos/MS (TsT) e Rochedo/MS (TsR), mostradas nas Figuras 4 A, B e C, foram observados padrões de coeficiente angular similares a população de referência.

Todavia, os valores do coeficiente angular das populações de triatomíneos dos municípios de Douradina/MS (TsD) e Aparecida do Taboado/MS (TsA), mostrados nas Figuras 4D e 4E, quando comparados à população de referência, apontaram menor homogeneidade e maior frequência de indivíduos com alelos de resistência, os quais podem ser selecionados futuramente, mediante forte pressão de inseticidas químicos.

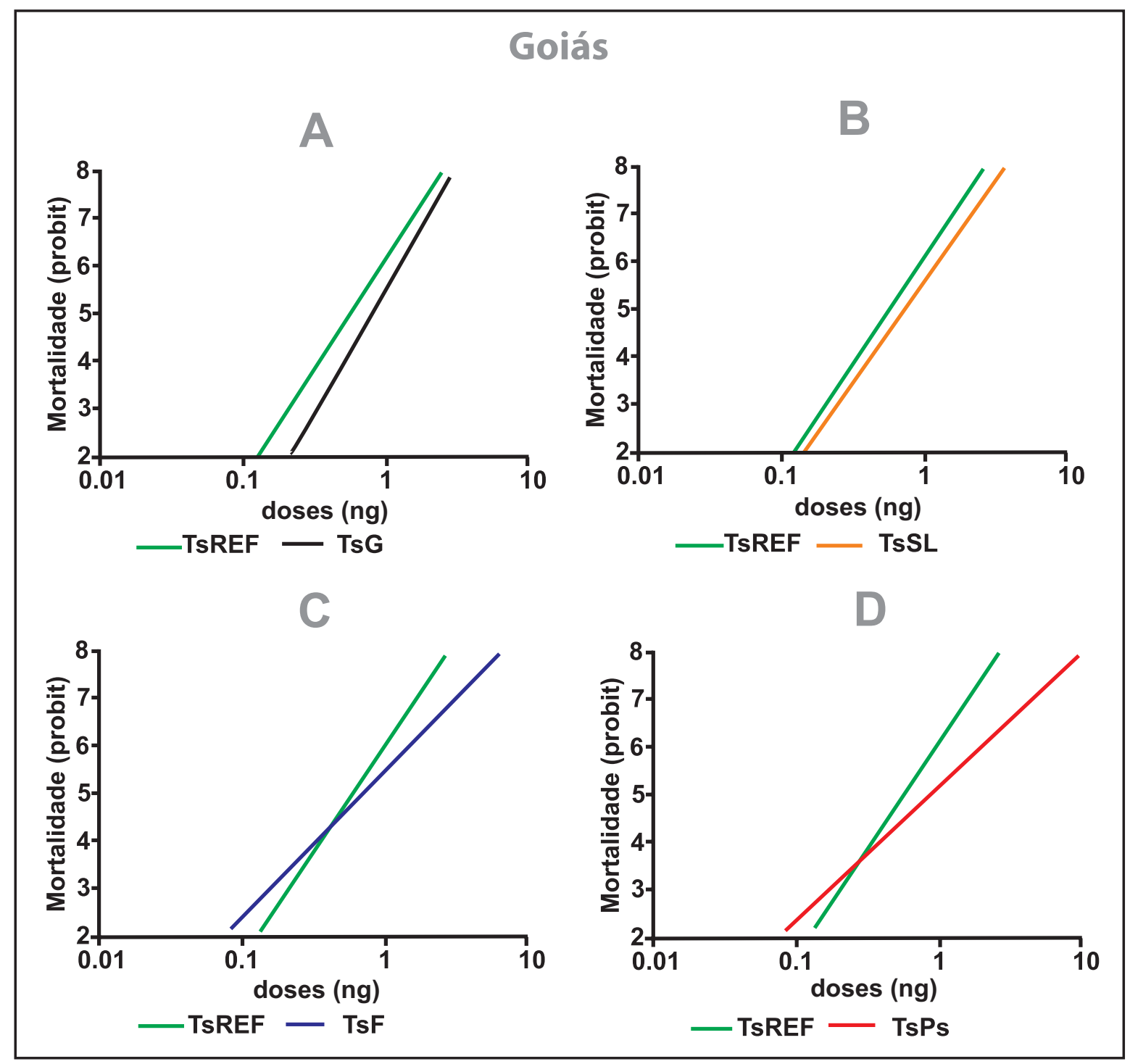

FIGURA 2 - Curva de mortalidade de populações de Triatoma sordida, em escala logarítmica, para o inseticida deltametrina. A: comparação entre TsREF $(4,51)$ e TsG $(5,36)$, B: comparação entre TsREF $(4,51)$ e TsSL $(4,18)$, C: comparação entre TsREF $(4,51)$ e TsF $(3,12)$, D: comparação entre TsREF $(4,51)$ e TsPs $(2,89)$.

TsREF: população de referência, TsG: Guarani de Goiás/GO, TsSL: São Luís de Montes Belos/GO, TsF: Firminópolis/GO, TsPs: Posse/GO. 


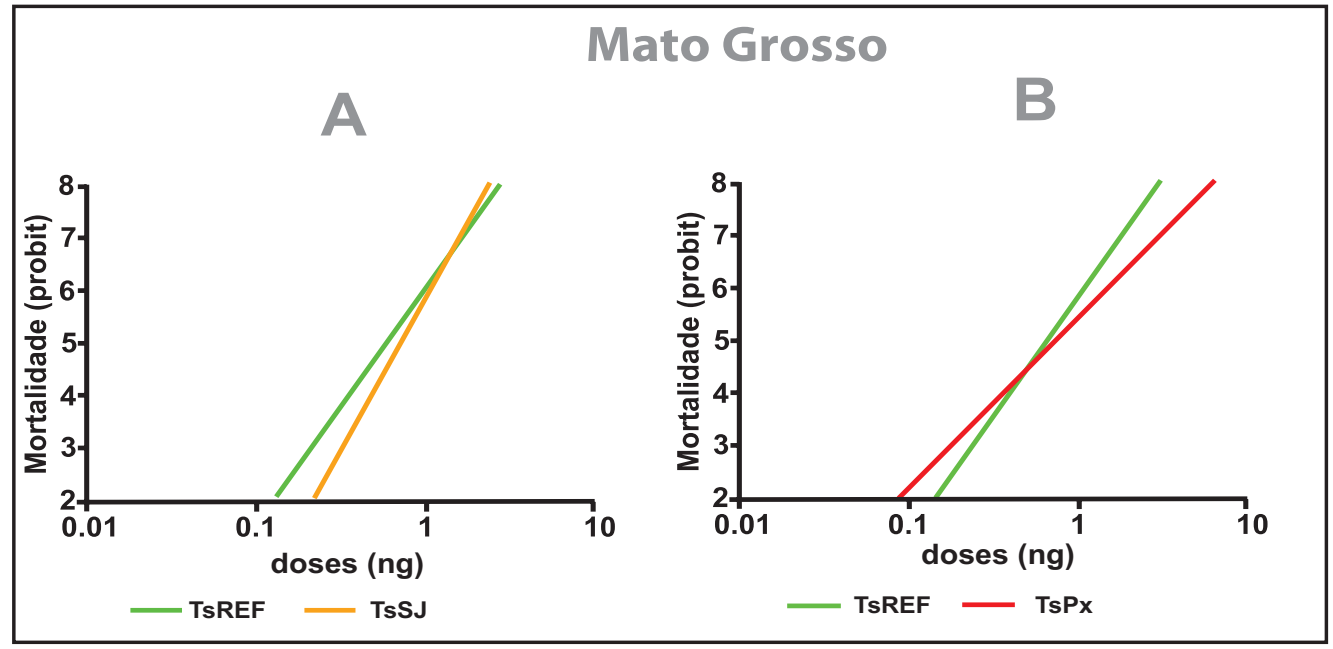

FIGURA 3 - Curva de mortalidade de populações de Triatoma sordida, em escala logarítmica, para o inseticida deltametrina. A: comparação entre TsREF $(4,51)$ e TsSJ $(5,72)$, B: comparação entre TsREF $(4,51)$ e TsPx $(3,25)$.

TsREF: população de referência, TsSJ: São José do Povo/MT, TsPx: Poxoréu/MT.

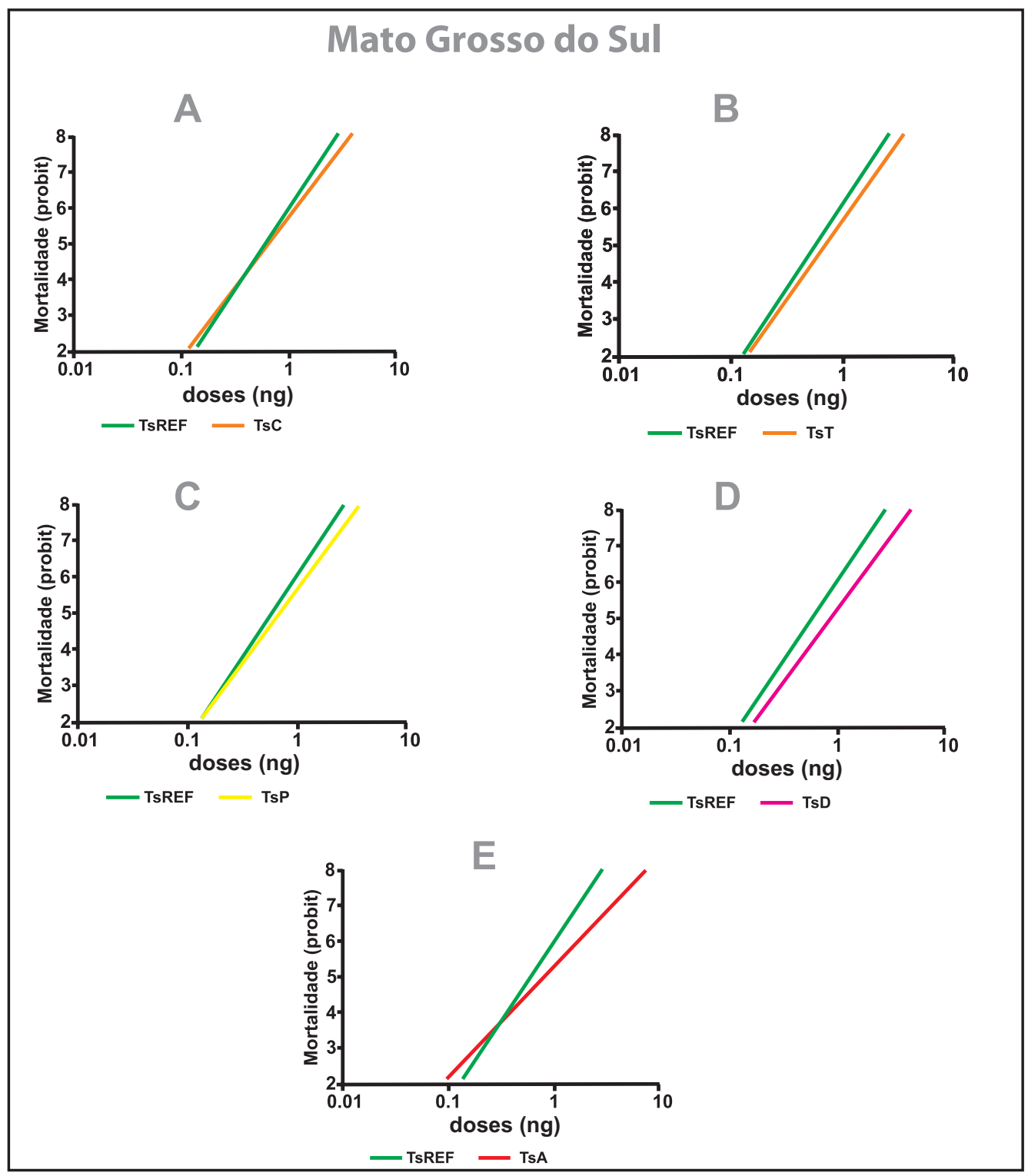

FIGURA 4 - Curva de mortalidade de populações de Triatoma sordida, em escala logarítmica, para o inseticida deltametrina. A: comparação entre TsREF $(4,51)$ e TsC $(3,95)$, B: comparação entre TsREF $(4,51)$ e TST $(4,27)$, C: comparação TsREF $(4,51)$ e TsR (4,23), D: comparação entre TsREF $(4,51)$ e TsD $(4,05)$, E: comparação entre TsREF $(4,51)$ e TsA $(3,15)$. TsREF: população de referência, TsC: Campo Grande/MS, TsT: Terenos/MS, TsR: Rochedo/MS, TsD: Douradina/MS, TsA: Aparecida do Taboado/MS. 


\section{DISCUSSÃo}

Os parâmetros de $\mathrm{RR} \leq 5$ para monitoramento de resistência de triatomíneos consideram que as diferenças de suscetibilidade são produzidas por variações individuais, ou seja, a resistência não está estabelecida, portanto as ações de controle devem prosseguir com o mesmo inseticida que está sendo utilizado ${ }^{22}$.

De acordo com Pessoa ${ }^{17}$, no Estado de Minas Gerais, há registros de cinco populações de $T$. sordida com níveis de resistência incipiente para deltametrina que demonstraram $\left.\mathrm{RR}_{50} \geq 5: \mathrm{I}\right)$ município de Coração de Jesus, Fazenda Domingada $\left(\mathrm{RR}_{50}=5,4\right)$; II $)$ município de Coração de Jesus, Fazenda Barriguda $\left(\mathrm{RR}_{50}=6,8\right)$; III $)$ município de Bocaiúva, Fazenda Chaves $\left.\left(\mathrm{RR}_{50}=5,8\right) ; \mathrm{IV}\right)$ município de Bocaiúva, Fazenda Félix I $\left(\mathrm{RR}_{50}=\right.$ $6,2) ; \mathrm{V})$ município de Presidente Juscelino $\left(\mathrm{RR}_{50}=5,5\right)$. Esse autor obteve um valor de $\mathrm{DL}_{50}$ igual a $0,065 \mathrm{ng}$ i. a./ninfa para a população de referência procedente de Uberaba/MG

Por outro lado, os resultados deste trabalho apontaram para a $\mathrm{DL}_{50}$ maior, cujo valor foi de $0,58 \mathrm{ng} /$ inseto. A população de referência demonstrou baixo nível de suscetibilidade, podendo ser considerada, até mesmo resistente, quando comparada com a utilizada por esse autor.

Ressalta-se que os parâmetros de RR são calculados a partir dos valores da $\mathrm{DL}_{50}$ da população de referência, consequentemente a variabilidade desses valores poderá interferir nos resultados de monitoramento e nas tomadas de decisões feitas pelo Programa Nacional de Controle da Doença de Chagas para o manejo de inseticidas, caso haja resistência estabelecida.

Por exemplo, nos resultados obtidos, neste trabalho, todas as populações foram classificadas como suscetíveis, conforme critérios estabelecidos pela Organização Pan-Americana de Saúde ${ }^{22}$. Os valores de $\mathrm{RR}_{50}$ foram menores que dois, utilizando a população de referência de T. sordida (TsREF) com $\mathrm{DL}_{50}=0,58 \mathrm{ng} /$ inseto.

Contudo, com utilização da linhagem suscetível de Pessoa ${ }^{17}$, cujo valor foi de $\mathrm{DL}_{50}=0,065 \mathrm{ng}$ i.a./ninfa as amostras seriam classificadas como resistentes, inclusive com recomendações de troca de inseticidas para algumas populações.

A utilização de duas ou mais populações de referência dificulta a interpretação dos limiares de RR. Para evitar possíveis erros na análise dos dados, sugere-se a padronização de população de referência para o monitoramento de resistência de triatomíneos.

Neste trabalho, a população utilizada como linhagem de referência (TsREF), foi obtida no início da década de 80. Assim, muito embora, ela tenha se originado de ambiente nunca exposto ao tratamento de inseticidas, a população fundadora dessa população pode ter chegado ao laboratório com presença de indivíduos resistentes.

Para este trabalho, utilizou-se população de referência procedente do município de Cordeiros, no Estado da Bahia. No passado, essa área pode ter sido submetida à forte pressão de diferentes classes de inseticidas, principalmente para o controle de T. infestans, assim como de outros insetos transmissores de doença ao homem. Nesse contexto, ressalta-se que a nossa linhagem de referência, talvez tenha sido isolada já com alguns indícios de resistência.

Em resumo, as estimativas das razões de resistência atribuídas para as onze populações de campo de T. sordida apontaram elevadas taxas de mortalidade. Os resultados foram similares aqueles verificados para populações de T. infestans procedentes dos municípios de
Doutor Maurício Cardoso $\left(\mathrm{RR}_{50}=0,72\right)$, Guarani das Missões $\left(R_{50}=0,97\right)$, Mato Queimado $\left(R_{50}=1,47\right)$ e Três de Maio $\left(\mathrm{RR}_{50}=1,86\right)$, Estado do Rio Grande do Sul, Brasil, as quais foram caracterizadas como sendo suscetíveis a deltametrina ${ }^{23}$.

No Brasil, o sucesso das campanhas de controle contra o principal vetor da DC, T. infestans, culminaram na certificação internacional de eliminação da transmissão da doença de Chagas pelo T. infestans, em junho de 2006.

A aplicação de inseticidas visando à eliminação de populações intradomiciliares e peridomiciliares de T. infestans, aparentemente, tem ocasionado grande impacto no controle de populações sinantrópicas de T. sordida.

Recentemente, os estudos de biologia molecular com T. sordida de áreas do cerrado (municípios de Espinosa e Mamonas), da Caatinga (município de Januária) e da Floresta do Paraná (município de Corinto) mostraram baixos níveis de variação genética em populações peridomésticas, o que poderia favorecer o modo de ação dos inseticidas utilizados nas campanhas de controle da $\mathrm{DC}^{24}$.

As cinco populações de triatomíneos dos municípios de Firminópolis/GO (TsF), Posse/GO (TsPs), Poxoréu/MT (TsPx), Douradina/MS (TsD) e Aparecida do Taboado/MS (TsA) apresentaram maior probabilidade de evolução de resistência quando comparadas com a população de referência (TsREF). Dessa maneira, as alterações temporais de suscetibilidade deverão ser rigorosas e, continuamente, acompanhadas para orientar adequadamente o planejamento das estratégias de controle.

Conclui-se que o monitoramento de resistência de populações nativas de T. sordida foi capaz de detectar pequenas alterações de resposta na taxa de suscetibilidade a deltametrina demonstrando baixos níveis de resistência $\left(R_{50}<2\right)$, nos onze municípios de 3 estados avaliados. Recomenda-se a definição de uma única população de referência de suscetibilidade para $T$. sordida e outras espécies a serem estudadas, assim como a adesão de outros laboratórios com infraestrutura e capacidade técnico-científica adequada para executar os bioensaios biológicos e bioquímicos, fornecendo subsídios para um melhor entendimento dos mecanismos de resistência e sua evolução.

\section{AGRADECIMENTOS}

À Dra. Claudia Vassena, do Centro de Investigaciones en Plagas e Insecticidas, Buenos Aires, Argentina, pela disponibilização das metodologias empregadas nos ensaios biológicos. À Grasiela D `Ávila Pessoa, do Centro de Pesquisas René Rachou/FIOCRUZ pelas sugestões das doses aplicadas nos bioensaios. Aos colegas Carmo Pereira, Mardones da Costa Flores Sobrinho, Kátia Maria Leal Braga, Karina Cavalcante e Joyce Pereira, do Laboratório de Entomologia Médica da CGLAB/SVS/MS, pelo auxílio na criação e manutenção do insetário de triatomíneos. Aos funcionários Aécio de Paula e Shirley R. Ramos, da Secretaria Estadual de Saúde do Mato Grosso, pela viabilização das capturas de triatomíneos.

\section{CONFLITO DE INTERESSE}

Os autores declaram não haver nenhum tipo de conflito de interesse no desenvolvimento do estudo. 


\section{REFERÊNCIAS}

1. Toledo MJO, Kuhl JB, Silva SV, Gasperi MV, Araújo SM. Estudo sobre triatomíneos e reservatórios silvetres de Trypanosoma cruzi no Estado do Paraná, Sul do Brasil. Resultados preliminares. Rev Soc Bras Med Trop 1997; 30:197-203.

2. Pires HHR, Borges EC, Andrade RE, Lorosa ES, Diotaiuti L. Peridomiciliary infestation with Triatoma sordida Stal, 1859 in the county of Serra do Ramalho, Bahia, Brasil. Mem Inst Oswaldo Cruz 1999; 94:147-149.

3. Oliveira AWS, Silva IG. Distribuição geográfica e indicadores entomológicos de triatomíneos sinantrópicos capturados no Estado de Goiás. Rev Soc Bras Med Trop 2007; 40:204-208.

4. Diotaiuti L, Azeredo BVM, Busek SCU, Fernandes AJ. Controle do Triatoma sordida no peridomicílio rural do município de Porteirinha, Minas Gerais, Brasil. Rev Panam Salud Publica 1998; 3:21-25.

5. Forattini OP, Rocha e Silva EO, Ferreira OA, Rabello EX, Pattoli D. Aspectos ecológicos da Tripanossomíase Americana: III - Dispersão local de triatomíneos, com especial referência a Triatoma sordida. Rev Saude Publica 1971; 5:193-205.

6. Diotaiuti L, Loiola CF, Dias JCP. The ecology of Triatoma sordida in natural environments in two different regions of the state of Minas Gerais, Brasil. Rev Inst Med Trop Sao Paulo 1993; 35:237-245.

7. Dias JP, Bastos C, Araújo E, Mascarenhas AV, Netto EM, Grassi F, et al. Acute Chagas disease associated with oral transmission. Rev Soc Bras Med Trop 2008; 41:296-300.

8. Dias JCP. Controle da Doença de Chagas. In: Dias JCP, Coura JR, organizadores. Clínica e terapêutica da Doença de Chagas. Uma abordagem para o clínico geral. Rio de Janeiro: Fiocruz; 1997. p.453-467.

9. Silveira AC. Enfoque de riesgo em actividades de control de triatominos. Rev Pat Trop 2004; 33:193-206.

10. Dias JCP, Macedo VO. Doença de Chagas. In: Coura JR, editor. Dinâmica das doenças infecciosas e parasitárias. Rio de Janeiro: Guanabara Koogan; 2005. v.1. p.557-593.

11. Vassena CV, Picollo MI, Zerba EN. Insecticide resistance in Brazilian Triatoma infestans and Venezuelan Rhodnius prolixus. Med Vet Entomol 2000; 14:51-55.

12. Vanessa CV, Picollo MI. Monitoreo de resistencia a insecticidas en poblaciones de campo de Triatoma infestans y Rhodnius prolixus, insectos vectores de la efermedad de Chagas. 2003. [acesso 12 maio de 2007]. Disponível em: http://www.sertox. com.ar/retel/default.htm/.

13. González-Audino P, Vassena C, Barrios S, Zerba E, Picollo MI. Role of enhance detoxication in a deltamethrin-resistant population of Triatoma infestans (Hemiptera, Reduviidae) from Argentina. Mem Inst Oswaldo Cruz 2004; 99:335-339.

14. Picollo MI, Vassena C, Orihuela PS, Barrios S, Zaidemberg M, Zerba E. High resistence to pyrethroid insecticides associated with ineffective field treatments in Triatoma infestans (Hemipetra: Reduviidae) from Northern Argentina. J Med Entomol 2005; 42:637-642.

15. Orihuela PLS, Vassena CV, Zerba EM, Picollo MI. Relative contribution of monooxygenase and esterase to pyrethroid resistance in Triatoma infestans (Hemiptera: Reduviidae) from Argentina and Bolivia. J Med Entomol 2008; 45:298-306

16. Toloza AC, Germano M, Cueto GM, Vassena C, Zerba E, Picollo MI. Differencial patterns of insecticide resistance in eggs and first instar of Triatoma infestans (Hemiptera: Reduviidae) from Argentina and Bolivia. J Med Entomol 2008; 45:421-426.

17. Pessoa GCA. Monitoramento da suscetibilidade ao piretróide deltametrina em populações de Triatoma sordida Stål, 1859 (Hemiptera: Reduviidae). [Dissertação de Mestrado]. [Belo Horizonte]: Centro de Pesquisas René Rachou da Fundação Oswaldo Cruz; 2008.95 p.

18. Lent H, Wygodzinsky P. Revision on the Triatominae (Hemiptera, Reduviidae) and their significance as vectors of Chagas'disease. Bull Am Mus Nat Hist $1979 ; 163: 123-520$.

19. World Health Organization. Protocolo de evaluación de efecto insecticida sobre triatominos. Acta Toxicol Argentina 1994; 2:29-32.
20. Lichfield J, Wilcoxon F. A simplified method of evaluation dose-effect experiments. J Pharmacol Exp Ther 1949; 96:99-113.

21. LeOra Software. Polo-PC: a user's guide to probit or logic analysis. Version 2.0 Berkeley, CA: LeOra Software; 2002.

22. Organização Pan Americana de Saúde. II Reunion tecnica latinoamericana de monitoreo de resistencia a insecticidas em triatominos vectores de Chagas. Panamá, 11 a 13 de abril de 2005.

23. Sonoda IV, Pessoa GCA, Cortez MR, Dias JCP, Romanha AJ, Diotaiuti L. Susceptibility of Triatoma infestans to deltamethrin in Rio Grande do Sul, Brazil. Mem Inst Oswaldo Cruz 2009; 104:668-670.

24. Monteiro FA, Jurberg J, Lasoki C. Very low levels of genetic variation in natural peridomestic populations of the Chagas disease vector Triatoma sordida (Hemiptera: Reduviidae) in Southeastern Brazil. Am J Trop Med Hyg 2009; 81:223-227. 